\title{
Probing the accretion disk - jet connection via instabilities in the inner accretion flow. From microquasars to quasars
}

\author{
Agnieszka Janiuk $^{1}$, Bożena Czerny ${ }^{2}$, Monika Mościbrodzka ${ }^{3}$, and \\ Aneta Siemiginowska ${ }^{4}$ \\ ${ }^{1}$ Center for Theoretical Physics, PAN, Al. Lotnikow 32/46, 02-668 Warsaw, Poland \\ email: agnieszka.janiuk@gmail.com \\ ${ }^{2}$ N. Copernicus Astronomical Center, Bartycka 18, 00-716 Warsaw, Poland \\ ${ }^{3}$ Department of Physics, University of Illinois, 1110 West Green Street, Urbana, \\ IL 61801, USA \\ ${ }^{4}$ Harvard Smithsonian Center for Astrophysics, 60 Garden St, Cambridge, MA 02138, USA
}

\begin{abstract}
We present various instability mechanisms in the accreting black hole systems which might indicate at the connection between the accretion disk and jet. The jets observed in microquasars can have a peristent or blobby morphology. Correlated with the accretion luminosity, this might provide a link to the cyclic outbursts of the disk. Such duty-cycle type of behaviour on short timescales results from the thermal instability caused by the radiation pressure domination. The same type of instability may explain the cyclic radioactivity of the supermassive black hole systems. The somewhat longer timescales are characteristic for the instability caused by the partial hydrogen ionization. The distortions of the jet direction and complex morphology of the sources can be caused by precession of the disk-jet axis.
\end{abstract}

Keywords. physical data and processes: accretion, X-rays:binaries, galaxies: active

\section{Overview}

Radiation pressure instability. The black hole accretion disk is subject to the thermal and viscous instability if the radiation pressure dominates over the gas pressure. If the accretion rate outside the unstable region (i.e. the mean accretion rate) is such that the
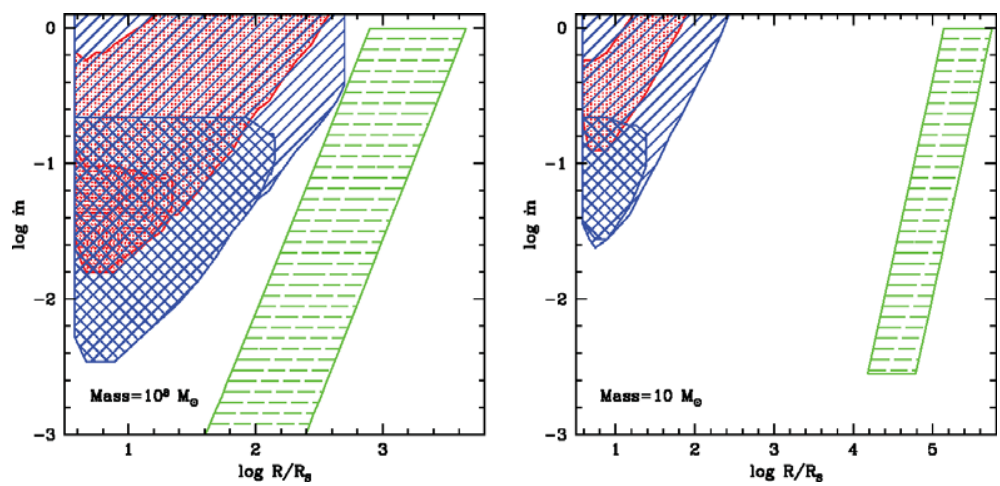

Figure 1. Extension of the radiation pressure (solid and dotted lines) and hydrogen ionization (dashed lines) unstable zones, depending on mean accretion rates (Eddington units). The results are for two heating prescriptions, as well as a zero and non-zero fraction of jet power. 
disk is unstable, the source enters a cycle of bright, hot states, separated by the cold, low luminosity states. The outburst amplitudes and durations are sensitive to black hole mass, viscosity and the mean accretion rate. If the viscous heating is proportional to the total pressure, the outburst amplitudes are very large - they can be reduced if the heating is proportional to the square root of the gas times the total pressure.

Partial Hydrogen ionization instability. Another type of the thermal - viscous instability is due to the partial hydrogen ionization. The disk also cycles between the two states: a hot and mostly ionized state with a large local accretion rate and a cold, neutral state with a low accretion rate. This instability has originally been proposed to explain the luminosity variations observed in cataclysmic variables. The same mechanism is responsible for the eruptions in soft X-ray transients (see e.g., Lasota (2001) for review) as well as may operate in active galactic nuclei (e.g., Janiuk et al. (2004)).

Disk precession. As studied by Janiuk et al.(2009), the acoustic instabilities of the Papaloizou-Pringle type can arise in the supersonic inner parts of the accretion flow. The azimuthal modes of such instabilities will lead to the tilt of the disk and its precession.

\section{Implications}

We speculate that in the hot state, the luminous core will power a radio jet, while during the cold state the radio activity ceases. In case of the regular periodic outbursts of GRS 1915+105 (see e.g. Fender \& Belloni (2004)), lasting from $\sim 100$ to $\sim 2000 \mathrm{~s}$, this approach is successful (e.g. Janiuk et al. (2002)). Only the limit cycle mechanism (likely driven by the radiation pressure instability) explains the absence of the direct transitions from its state $\mathrm{C}$ to the state $\mathrm{B}$, as well as the observed QPO oscillations. Scaling the timescale with the black hole mass by a factor $10^{8}$ gives the outbursts durations of $10^{2}-10^{4} \mathrm{yrs}$, and amplitudes are sensitive to the energy fraction deposited in the jet (see Fig. 1). The radiation pressure instability operating above $\dot{m}=0.025$ may be responsible for very short ages of Compact Symmetric Objects (Czerny et al. (2009)). The radiostructures may be influenced by jet precession (Kunert-Bajraszewska et al.(2010))

In the ionization instability, the derived separations between outbursts are on order of $10^{6}$ yrs for a $10^{8} M_{\odot}$ black hole, while the outburst duration is an order of magnitude shorter. The location of the unstable zone is sensitive to the black hole mass (see Fig. 1). For an X-ray binary, the disk maximum radius is limited by the Roche-lobe size and might be too small for an appropriate temperature range to appear. In AGN, the partly ionized zone is much closer to the black hole. If the two unstable zones are very close to each other, the rate of supply of material to the radiation pressure dominated region may be modulated on long timescales, independently of the environment in the host galaxy.

\section{Acknowledgements}

This work was supported in part by grant NN 203512638 from the Polish Ministry of Science.

\section{References}

Czerny, B., et al. 2009, ApJ, 698, 840

Fender, R. \& Belloni, T. 2004, ARAA, 42, 317

Janiuk, A., Czerny, B., \& Siemiginowska, A. 2002, ApJ, 576, 908

Janiuk, A., Czerny, B., Siemiginowska, A., \& Szczerba, R., 2004,ApJ, 602, 595

Janiuk, A., Sznajder, M., Moscibrodzka, M., \& Proga, D. 2009, ApJ, 705, 1503

Kunert-Bajraszewska, M., Janiuk, A., Gawronski, M., \& Siemiginowska, A. 2010, ApJ, 718, 1345

Lasota, J.-P., 2001, New Astron. Revs., 45, 449 